\title{
Morphological Investigations of Electrochemically Deposited Polyaniline Films
}

\author{
A. G. Bedekar, ${ }^{*}$ S. F. Patil, ${ }^{*}$ R. C. Patil, ${ }^{*}$ and Chitra Agashe** \\ * Department of Chemistry, University of Poona, Pune 411007, India \\ ** Department of Physics, University of Poona, Pune 411007, India
}

(Received May 12, 1994)

\begin{abstract}
The effect of rate of mass transfer on the surface morphology of electrochemically deposited polyaniline films was studied using scanning electron microscopy (SEM). The rate of mass transfer was controlled by varying the sheet resistance of electrode and the monomer concentration. Both these parameters are found to affect the deposition process and the surface morphology to a considerable extent. The polyaniline film deposited using $0.1 \mathrm{M}$ monomer concentration on electrode with $50 \Omega / \square$ sheet resistance shows a granular structure joined together with fine fibrils. Increase in the monomer concentration and decrease in the sheet resistance of the electrode lead to the enhancement in the growth of the polymer film as revealed from the current time transient measurements. The formation of fibres with different shapes such as rope-like, curved, cyclone-like, petal-like, wavy, wing-like, and criss-cross linking depends on the growth conditions of films. The sheet resistance of electrode mainly determines the number density of these fibres, whereas, the patterning is governed by the monomer concentration.

KEY WORDS Polyaniline / Electrochemical Deposition / Morphology / Scanning Electron Microscopy (SEM) /
\end{abstract}

Polyaniline has turned out to be a very promising electroconductive polymeric material because of its ease in preparation as well as good environmental stability. Attempts are being made to prepare thin films of this material having good thermal, mechanical and electrical properties so as to use these films for various applications as in electrochromic devices, batteries, smart windows etc. ${ }^{1,2}$ For such kind of applications it is important that the surface of the film be quite smooth. Therefore, it becomes necessary to study the morphological properties of films deposited under various experimental conditions. This would help in establishing the optimum conditions for obtaining good quality films. Limited work in this regard have been published. ${ }^{3-5}$

We have already reported that the sheet resistance of the electrode controlls the rate of deposition of poly $(o$-anisidine $)$ films, while the monomer concentration determines the extent of formation of variable oxidation states of polymer. ${ }^{6}$ The present study was undertaken to examine the effect of rate of mass transfer on the morphological properties by varying sheet resistance of electrode and monomer concentration on the electrochemically deposited polyaniline films using SEM technique.

\section{EXPERIMENTAL}

All the chemicals used were of analytical reagent grade. The aqueous solutions were prepared using double distilled water. Transparent $\mathrm{SnO}_{2}: \mathrm{F}$ thin films having coated on corning 7059 glass substrates were used as electrodes. Electrodes with three different sheet

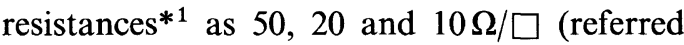

*1 The sheet resistance, $\rho_{\mathrm{s}}(\Omega / \square)$ is defined as $\rho_{\mathrm{s}}=\rho / t$ where $\rho$ is the reciprocal of conductivity $(\Omega \mathrm{cm})$ and $t$ is the thickness of, film (cm) (c.f., Dieter K. Schroder, "Semiconductor Material and Device Characterization," John Wiley \& Sons, New York, N.Y., 1990). 


\section{A. G. Bedekar et al.}

to as type $\mathrm{A}, \mathrm{B}$, and $\mathrm{C}$, respectively) were obtained by spraying different volumes of the solution. The details of which are described in our earlier paper. ${ }^{7}$

The polyaniline films were deposited electrochemically under potentiostatic conditions using a potentiostat/galvanostat [Model CL95 Elico Pvt. Ltd., India] at an applied voltage of $1.6 \mathrm{~V}$ for a time interval of $3 \mathrm{~min}$. Electrolyte solutions with different concentrations of aniline monomer were prepared by adding appropriate volumes of aniline to $1.0 \mathrm{M} \mathrm{HCl}$. Identical set of electrodes were used as cathode and anode while SCE was used as the reference electrode. After deposition, the films

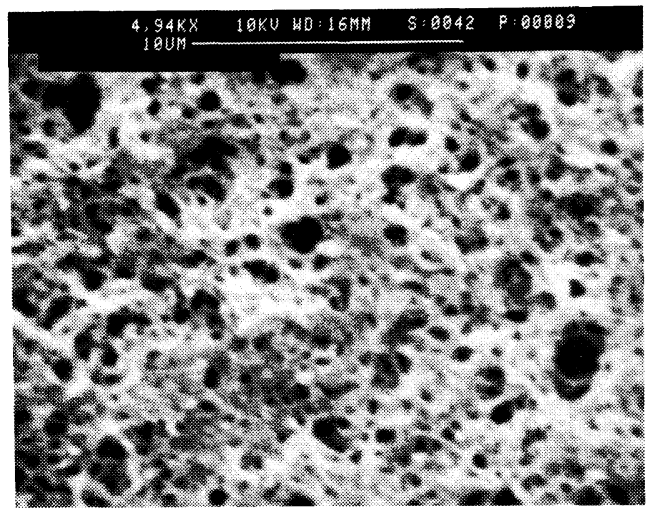

(a)

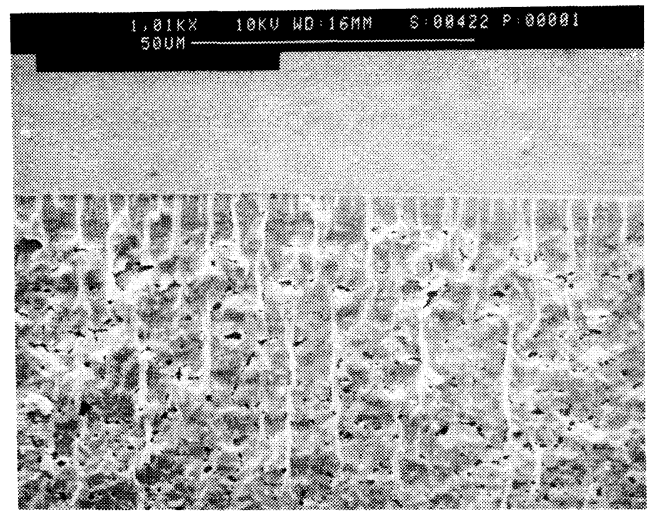

(b) were washed with water, dried and used for obtaining the micrographs using a scanning electron microscope (Stereoscan S 120, Cambridge Instruments Ltd., U.K.). Before taking the micrograph the films were coated with a thin layer of gold (thickness $\sim 20 \mathrm{~nm}$ ) using a sputter coater unit (E 5000 BioRad, U.K.).

\section{RESULTS AND DISCUSSIONS}

The deposited films were deep green in colour, soft to touch and slightly non adherent. The softness might be due to the fibrillar type of growth as discussed below.

Figure 1(a)-(d) show the scanning electron

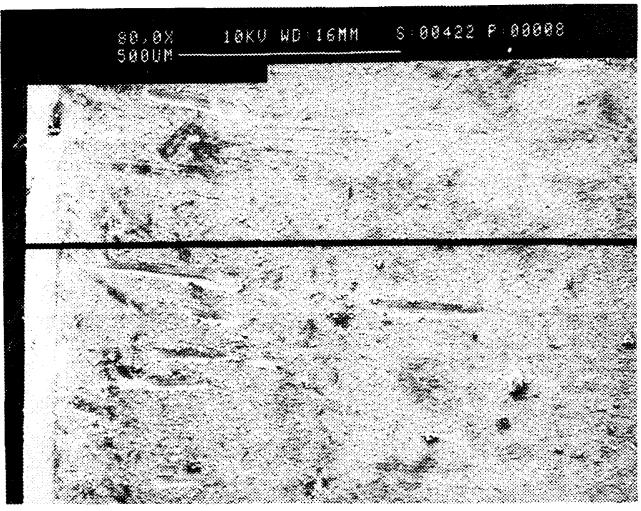

(c)

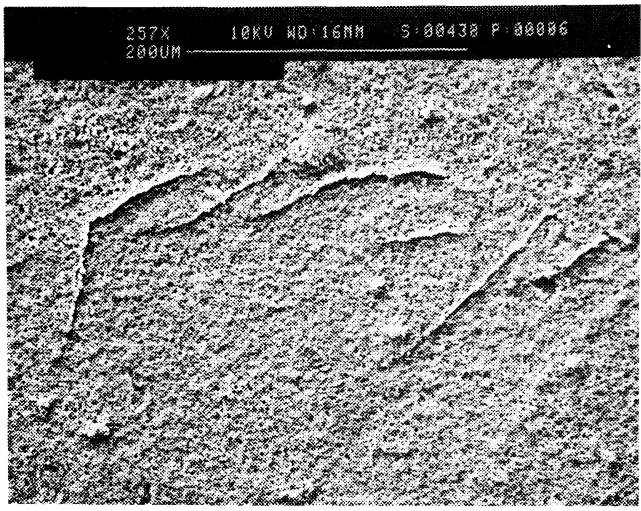

(d)

Figure 1. SEM's of polyaniline films deposited in presence of $0.1 \mathrm{M}$ monomer concentration on (a) A type electrode exhibiting net-like structure; (b) B type electrode exhibiting rope-like fibres; (c) B type electrode exhibiting bar-like straight fibres; (d) $\mathrm{C}$ type electrode exhibiting rare distribution of thick individual fibres. 
micrographs of electrochemically deposited polyaniline films as a function of sheet resistance of electrode at $0.1 \mathrm{M}$ monomer concentration. No specific pattern of morphology was observed for the film grown on type A electrode (Figure 1(a)). This micrograph shows formation of a net-like structure which consists of small granules joined together with interconnecting channels that look like fibrils. Similar observations had also been reported by earlier authors. ${ }^{3-5}$ On type $B$ electrode one could observe the peculiar formation of thin parallel rope-like conducting channels developed densely at the surface of the film (Figure 1(b)). The thickness of these fibrils is found to be $\sim 1.5 \mu \mathrm{m}$ and the length $\sim 25 \mu \mathrm{m}$. It is observed that the density of the fibres decreases at the bottom edges of the electrodes (Figure 1(c)). A few straight fibres having a thickness of $\sim 50 \mu \mathrm{m}$ extending up to a length of around $350 \mu \mathrm{m}$ are also observed at these edges. In the case of film grown on type $\mathrm{C}$ electrode (Figure 1(d)) a rare distribution of individual fibres about $6-20 \mu \mathrm{m}$ thick and above $125 \mu \mathrm{m}$ in length are seen towards the edges of the electrode.

These results can be explained by considering the morphology of $\mathrm{SnO}_{2}: \mathrm{F}$ films. The films with higher sheet resistance of $50 \Omega / \square$ (A type electrodes) exhibit a uniform morphology with $\mathrm{SnO}_{2}: \mathrm{F}$ grains having a preferred orientation along [200] direction. In contrast to this, $\mathrm{SnO}_{2}: \mathrm{F}$ films with a sheet resistance of $10 \Omega / \square$ (C type electrodes), show a reorientational effect in the upper layers. In other words the $\mathrm{SnO}_{2}: \mathrm{F}$ grains are arranged in different directions other than [200] direction. ${ }^{8}$ Due to this, the latter would provide greater fraction of nucleation centres for polymer growth in comparison with the former, and lead to the higher deposition rate thereby affecting the morphology of the polymer film. The authors have to mention that the $\mathrm{SnO}_{2}: \mathrm{F}$ films used did not show any irregularities like cracks or scratches as these are known to affect the growth process of the polymer film. ${ }^{9}$ The

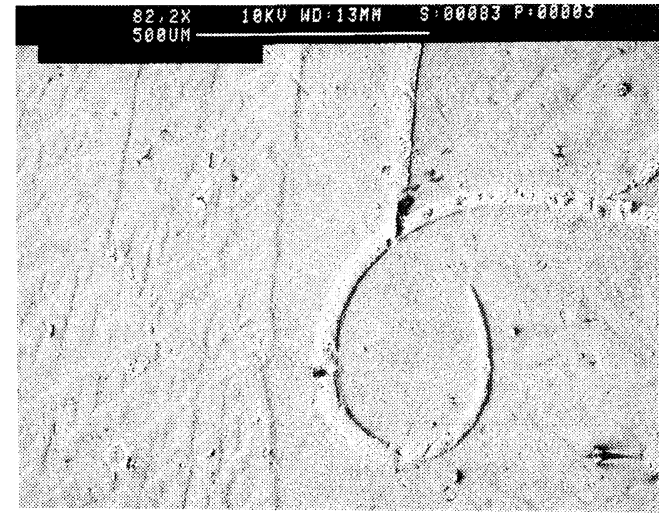

(a)

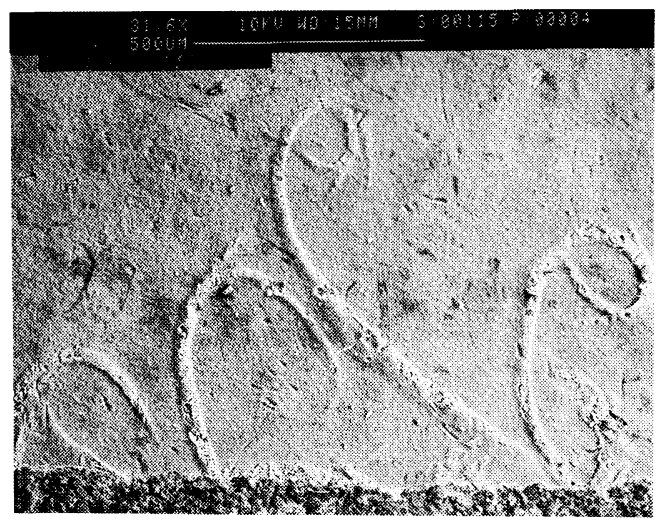

(b)

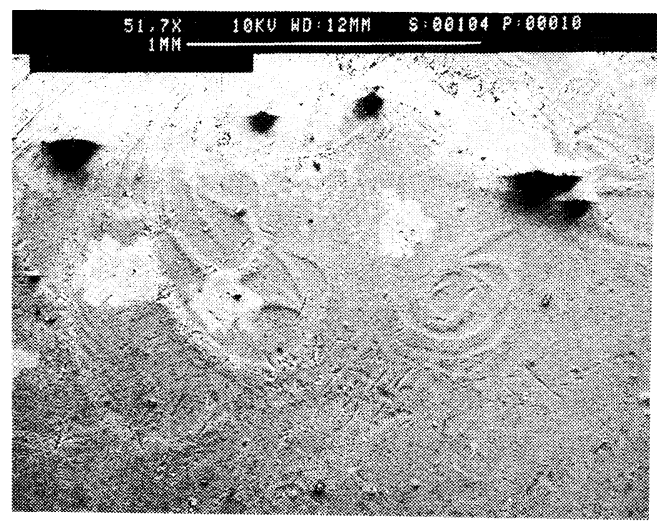

(c)

Figure 2. SEM's of polyaniline films deposited in presence of $0.2 \mathrm{M}$ monomer concentration on (a) A type electrode exhibiting a mixture of curved and straight fibres; (b) B type electrode exhibiting a family of curved fibres; (c) C type electrode exhibiting cyclone like structure. 


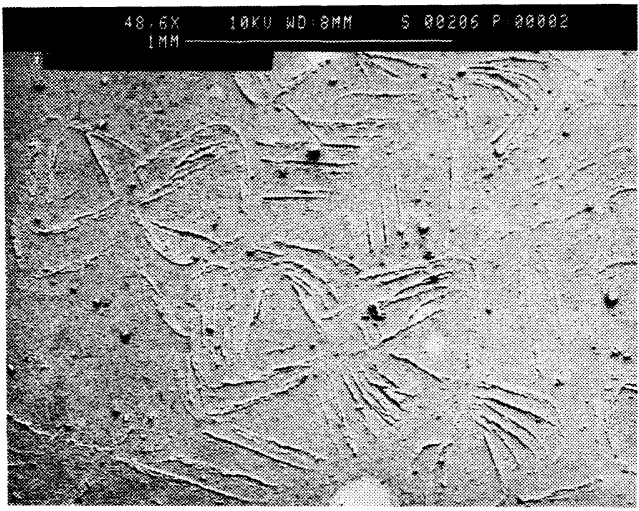

(a)

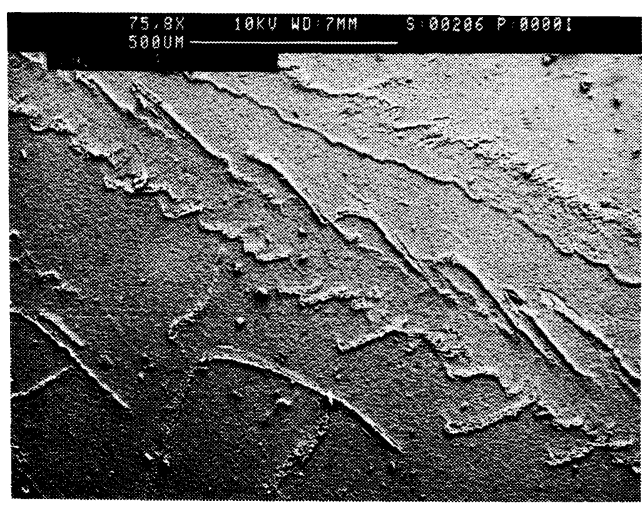

(b)

Figure 3. SEM's of polyaniline film exhibiting the formation of (a) petal like fibres, (b) wave like fibres [monomer concentration $=0.4 \mathrm{M}$, A type electrode].

growth of the film on type A electrode is so slow that only a thin layer of the deposit with considerable homogeneity is obtained leading to no specific pattern. As we go to type $B$ electrode with intermediate sheet resistance the nucleation centres and consequently the current density would increase appreciably, enhancing the growth rate of the polymer. Once a thin layer of polymer is formed, further layers would grow depending on the arrangement of the molecules in the previous layer. The growth of thin rope-like structures may arise due to the higher deposition rate caused by the additional gaseous species at the air-electrolyte interface. On the contrary, the absence of such

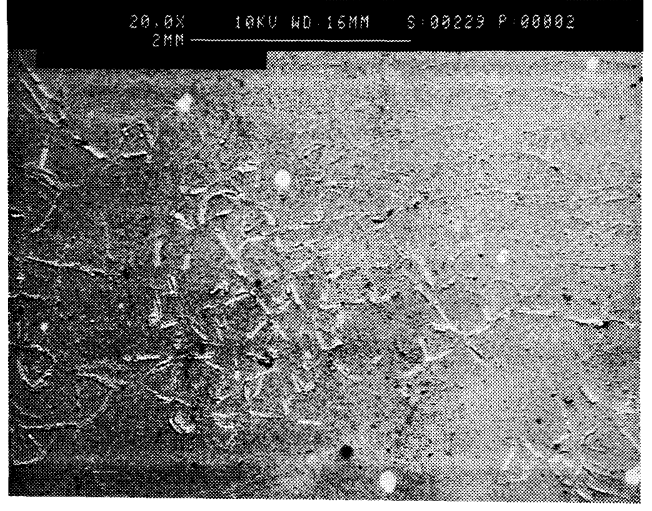

(a)

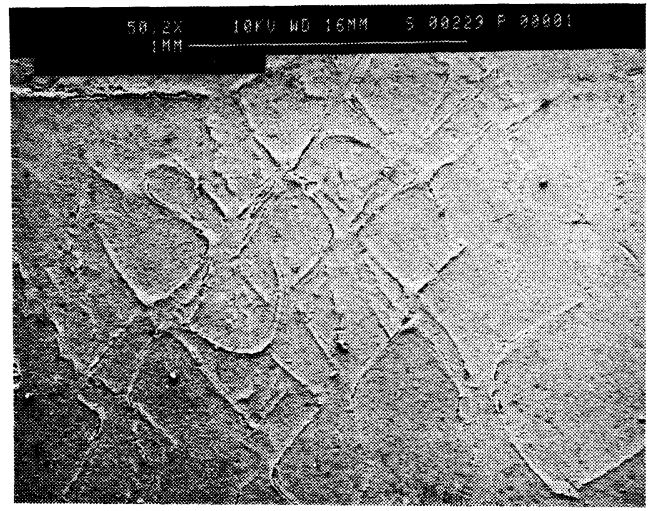

(b)

Figure 4. SEM's of polyaniline film exhibiting formation of (a) wing like fibres, (b) magnified picture of wing like patterning [monomer concentration $=0.4 \mathrm{M}, \mathrm{B}$ type electrode].

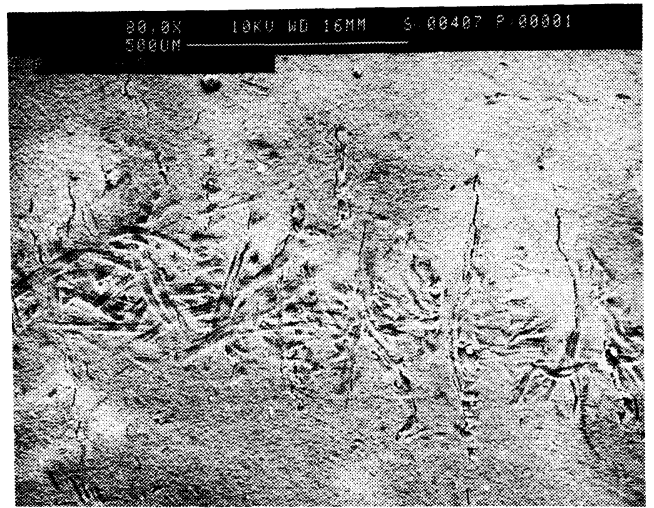

Figure 5. SEM of polyaniline film exhibiting criss-cross mixing of fibres [monomer concentration $=0.4 \mathrm{M}, \mathrm{C}$ type electrode]. 
species in the bulk of the electrolyte allows the formation of thicker, straight bar-like fibres with a lower density. Further, the accelerated deposition rate on type $\mathrm{C}$ electrode lead to the formation of thicker films and hence multiple layers are expected to be deposited on these electrodes. As a result, thicker and longer fibres, although fewer in number are observed in this case (Figure 1(d)).

The micrographs of polyaniline films grown on electrodes of different sheet resistances in the presence of $0.2 \mathrm{M}$ concentration of monomer in the electrolyte are depicted in Figure 2(a)-(c). In Figure 2(a), a mixture of curved and straight fibres grown at the edge of the electrode (type A) having an average thickness of $\sim 40 \mu \mathrm{m}$ are observed. The micrograph also shows the presence of striates. The film on type B electrode exhibits a family of curved fibres (Figure 2(b)) with a variable width ranging between $25-75 \mu \mathrm{m}$ having different lengths. Films grown on type $\mathrm{C}$ electrodes (Figure 2(c)) also show curved fibres, however, the curvature is so high that it forms a cyclone-like structure. These fibres have a typical width of $\sim 60 \mu \mathrm{m}$. Figure 2(a)(c) present clearly the exclusive formation of curved fibres which may arise due to the enhancement in the deposition rate because of use of higher concentration of monomer for the deposition of the films. On type A electrodes therefore, one can observe few fibres while the number increases as we go to type $\mathrm{B}$ and $\mathrm{C}$ electrodes. The increase in the curvature can be attributed to the addition of monomer ions to the developing fibres, instead of the growth starting, at a new point due to lack of time as a result of enhanced growth rate. This also contributes towards increasing the thickness of the fibre.

Figures 3-5 exhibit the morphological features of polyaniline films grown as a function of sheet resistance of electrode for a monomer concentration of $0.4 \mathrm{M}$ in the electrolyte solution. Figure 3 , reveals the presence of two types of joining of conducting
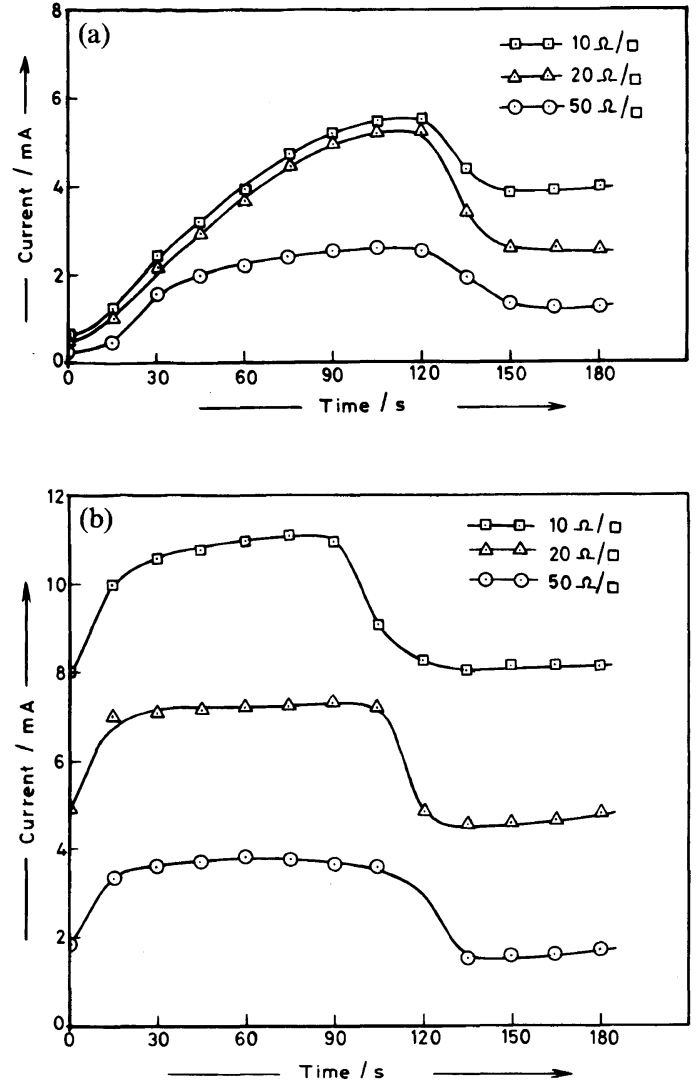

Figure 6. Current-time transients of polyaniline films obtained at (a) $0.1 \mathrm{M}$ and (b) $0.4 \mathrm{M}$ monomer concentration [deposition voltage $=1.6 \mathrm{~V}$ ] .

channels; petal-like (Figure 3a) and wave-like (Figure 3(b)) formation throughout the film on A type electrodes. The width of petal-like fibres is $\sim 30 \mu \mathrm{m}$, while that of wave-like fibres is $\sim 20 \mu \mathrm{m}$. The length of these fibres ranges between $300-500 \mu \mathrm{m}$. Yet another type of morphology is observed viz. a wing-like structure on type B electrode (Figure 4(a)) which is clearly seen at higher magnification (Figure 4(b)). The fibres having the width of about $30 \mu \mathrm{m}$ and length of $250 \mu \mathrm{m}$ are observed in this case. On type $\mathrm{C}$ electrodes the film shows criss-cross mixing of fibres at the surface of the electrode extending upto a certain length towards the center. A considerable tension is seen to develop on film layers finally leading 
to the development of cracks, seen as dark lines in the micrograph (Figure 5). For these set of films, again the growth would be accelerated due to further increase in monomer concentration. As a result, the growth can be seen not only at the edges as in the earlier two cases (at 0.1 and $0.2 \mathrm{M}$ monomer concentration, Figures 1 and 2), but almost throughout the film. A well defined patterning is observed and the fibres are seen to be interconnected through the curved portion. On type A electrode the distribution of fibres is rare as compared to those observed on type B electrodes where they are denser and thicker. On the contrary, on type $\mathrm{C}$ electrode the effect of monomer concentration is found to be too evident. It appears that there must be a forceful drag of the monomer ions towards the electrode surface from the solution. The monomer ions thus have no choice but to get attached randomly at any point on the previously grown layer of the fibre. A new fibre thus develops and the process continues leading to a large disturbance in the growth of the film which in turn damages the morphology of the film as a whole.

These results are confirmed from the nature of the current-time transients observed on type $\mathrm{A}, \mathrm{B}$, and $\mathrm{C}$ electrodes at 0.1 and $0.4 \mathrm{M}$ monomer concentrations (Figure 6). The rapid increase in the magnitude of current with decrease in the sheet resistance of the electrode at $0.1 \mathrm{M}$ monomer concentration is consistent with the faster nucleation process followed by saturation. While the sharp drop in current subsequently reaching steady state value can be attributed to the joining up of individual grains through interconnecting channels simultaneous to the growth of next layer. Similarly the shift in the initial saturation value along with the region of steep drop towards lower time scale observed at higher monomer concentration $(0.4 \mathrm{M})$ justifies the accelerated rate of deposition of polymer films.

\section{CONCLUSIONS}

The surface morphology of electrochemically deposited polyaniline films is strongly governed by the rate of mass transfer which in turn depends on sheet resistance of electrode and the monomer concentration. The sheet resistance of electrode determines the number density of different fibres resulting from the accelerated growth at particular sites. The patterning in the films is decided predominantly by the concentration of monomer used for deposition.

Acknowledgements. The authors (CA and RCP) are thankful to the CSIR for providing the fellowships. Thanks are also to $\mathrm{Mr}$. Rajdeep Dongre of Agharkar Research Institute, Pune, for taking the SEM micrographs and to Prof. H. J. Arnikar for his fruitful suggestions.

\section{REFERENCES}

1. M. G. Kanatzidis, Chem. Eng. News, Dec. 36 (1990).

2. S. Roth and W. Graupner, Synth. Met., $55-57,3623$ (1993).

3. Wan. Meixiang, Synth. Met., 31, 51 (1989).

4. Wu. S. Huang, B. D. Humphrey, and A. G. Mac Diarmid, J. Chem. Soc. Faraday Trans., 82(1), 2385 (1986).

5. M. Fujii, K. Arii, and K. Yoshino, J. Phys. Condens. Matter., 3, 7207 (1991).

6. A. G. Bedekar, S. F. Patil, R. C. Patil, and C. M. Agashe, J. Phys. D, Appl. Phys., 27, 1727 (1994).

7. C. Agashe, M. G. Takwale, B. R. Marathe, and V. G. Bhide, Sol. Energy Mater., 17, 99 (1988).

8. C. Agashe and B. R. Marathe, J. Phys. D, Appl. Phys., 26, 2049 (1993).

9. J. Lukkari, R. Tuomala, S. Ristimaki, and J. Kankare, Synth. Met., 47, 217 (1992). 